\title{
A System of Information Systems to Capitalize Resources of Collaborative Activities: the ECOPACK Project
}

\author{
Zoubida Afoutni \\ Sorbonne Universités, Université de \\ Technologie de Compiègne, CNRS \\ Heudiasyc UMR 7253 CS 60319 \\ 60203 Compiègne, France \\ zoubida.afoutni@utc.fr
}

\author{
Majd Saleh \\ Sorbonne Universités, Université de \\ Technologie de Compiègne, CNRS \\ Heudiasyc UMR 7253 CS 60319, \\ 60203 Compiègne, France \\ majd.saleh@utc.fr
}

\author{
Claude Moulin \\ Sorbonne Universités, Université de \\ Technologie de Compiègne, CNRS, \\ Heudiasyc UMR 7253 CS 60319, \\ 60203 Compiègne, France \\ claude.moulin@utc.fr
}

\author{
Véronique Misséri \\ Sorbonne Université, Université de \\ Technologie de Compiègne, CNRS, EA \\ 2223 Costech Compiègne, France \\ veronique.misseri@utc.fr
}

\author{
Marie-Hélène Abel \\ Sorbonne Universités, Université de \\ Technologie de Compiègne, CNRS, \\ Heudiasyc UMR 7253 CS 60319, \\ 60203 Compiègne, France \\ marie-helene.abel@utc.fr
}

\begin{abstract}
This paper describes a System of Information Systems (SoIS) to capitalize heterogeneous resources of collaborative activities in perspective of strategic analysis. The de-centralization of work processes have led organizations to respond to their dynamic environments and nomads practices by using collaborative software such as brainstorming environment. The outputs of a brainstorming are huge amounts of knowledge that need to be shared, capitalized and structured to generate a support for new discussions. While preparing, or during a brainstorming, participants may use other systems/web tools to gather more information. This information are external resources that improve the brainstorming efficiency and have to be capitalized in the same way as the knowledge produced during the brainstorming to "re-invent" the way of strategic analysis. It is therefore a matter of managing a set of resources generated by a set of independent systems. For this, we propose a solution based on SoIS approach. More precisely, we propose a solution to integrate a collaborative application called ECOPACK within a large SoIS called MEMORAeSoIS. ECOPACK is a digital ecosystem that meets the needs of ideation, innovation and strategic analysis. It offers several functionalities such as the capitalization of resources produced during a meeting. However, as mentioned above, participants may use resources coming from other systems. To allow capitalizing external resources, we use the MEMORAeSoIS that provides the ability to manage resources of autonomous systems. We illustrate our proposition by a scenario on the challenges of France's naval defense. This paper provides a solution based on the System of Information Systems approach to capitalize collaborative activities.
\end{abstract}

\section{CCS CONCEPTS}

-Information systems $\rightarrow$ Collaborative and social computing systems and tools; Wrappers (data mining); •Computer systems organization $\rightarrow$ Architectures;

\section{KEYWORDS}

System of Information Systems, Knowledge capitalization, Brainstorming Platform.

\section{INTRODUCTION}

The emerging of technological development and communication technologies has led to the emergence of new form of work known as collaborative work. Brainstorming environments play important role to enhance collaborative work in organization by providing a tool to the stakeholders allowing them generating innovative idea, increasing creative efficiency or finding solutions to different problems. During brainstorming activities, participants may use several systems and web tools with dedicated purposes to accomplish their tasks. Among this systems we can cite, systems to comment, to note and to capitalize resources of collaborative activities, project management software, social networks, wiki systems, etc. For example, a stakeholder that uses a TiddlyWiki to take some notes about the topic of the brainstorming while preparing it. From this point of view, participants in a brainstorming session use a set of resources coming from autonomous and heterogeneous systems to collaborate and accomplish their activities. To get these resources, participants have to query each system/web tools independently. This makes difficult the task of storage, sharing and capitalization of these resources. Also, the outputs of a brainstorming are huge amounts of knowledge that may be interesting for other projects, help to explain some decisions made during the brainstorming, etc. The question now is how to manage, to capitalize, to share, to reuse all 
resources used and produced in collaborative activities? To answer this question there is a need of an adequate architecture and a robust semantic information systems allowing gathering, representing and storing various heterogeneous information coming from different systems. We are interesting thus in capitalization and reuse heterogeneous resources of independent systems. More precisely, we are interesting in managing resources used and produced by a collaborative application called ECOPACK. It is a digital ecosystem that meets the needs of ideation, innovation and strategic analysis. To reach our goal, we use the System of Information Systems (SoIS) approach. In [10] authors propose a SoIS system called MEMORAeSoIS to manage heterogeneous resources of different system from a single point. MEMORAeSoIS architecture has the advantage to be flexible which facilitates adding a new system without an important development effort. We have chosen thus this platform to integrate ECOPACK platform in order to: (i) offer to users the possibility to access easily various system/web tools from a single point (ii) allow users capitalizing, sharing and reusing all resources used and produced during their collaborative activities.

This paper is organized as follows: section 2 presents the brainstorming collaborative activities and the System of Information Systems approach. In section 3, we present first ECOPACK and MEMORAeSoIS platforms then we describe a use case to illustrate our proposal and how ECOPACK has been integrated within MEMORAeSoIS. In section 4, a conclusion and future works are presented.

\section{LITERRATURE REVIEW}

\subsection{Brainstorming collaborative activities}

Brainstorming approach was coined by Osborn [8] who defines a set of rules aiming to stimulate ideation productivity. Brainstorming can be seen as a training process to stimulate cognitive processes for imagination and flexibility in creative thinking. Two kinds of brainstorming activities can be distinguished [7]: (i) participants are alone and prepare future collaborative sessions (ii) participants work during collaborative sessions involving one or more teams.

In the first case, even if participants are alone, their activities are considered as collaborative because they are accomplished for the benefit of a group of workers [7]. For example, people insert in a common repository a new document; annotate a document or a fragment of a document produced by others. In this type of activities participants use a tool designed for personal PCs or a Web application. While preparing a brainstorming session, participants use different Information System and Web tools. For example, a tool facilitating project management such as Trello ${ }^{1}$ or $\mathrm{Kanban}^{2}$ system. The set of systems and tools used while preparing a brainstorming session, produce a set of resources that are in major case stored on participants' PCs. To share these resources, participants have for example to use email system or

\footnotetext{
${ }^{1}$ https://trello.com/

${ }^{2} \mathrm{https} / / /$ kanbanize.com.
}

a file hosting service such as DropBox. However, there are no tools allowing capitalizing heterogeneous resources of different systems.

In the second case, during brainstorming sessions, people can progressively agree on some definitions and on some decisions. To this, participants use dedicated tools such as iMindMap ${ }^{3}$, or Mindmeister ${ }^{4}$. These tools allow users discussing, make notes, visualizing data of brainstorming session. However, once again discussions and argumentations may require the study of external resources that are not accessible from the brainstorming tools. Consequently, to carry out collaborative activities, it is necessary not only to have a tool to discuss with, to visualize data, to make comment but also a tool to access other useful systems, to share all resources, to keep track of external resources (from other systems) or the resources produced during brainstorming activities.

\subsection{System of systems approach}

The term System-of-Systems (SoS) is widely recognized and is often used to describe complex assembly of distributed standalone parts. Its application area spans from original military to other domains, especially system engineering. To give a common understanding of SoS concept and its characteristics, various efforts have been made. In [5], "An SoS is defined as a set or arrangement of systems that results when independent and useful systems are integrated into a larger system that delivers unique capabilities". The capability is the ability to achieve a desired effect. In [9] a SoS is a collection of systems tied together to create a more complex system. The resulting system is able to achieve a global mission that its subsystems alone cannot fulfill; a SoS has five key features [6]: (i) Operational independence of elements (ii) managerial independence of elements (iii) evolutionary development (iv) emergent behavior (v) geographical distribution.

In [11], authors go further in defining an evolutionary SoS by adding two principles: (i) the complexity of the SoS framework does not grow as constituent systems are added, removed, or replaced (ii) the constituent SoS do not need to be re-engineered as other constituent systems are added, removed, or replaced.

Also, according to the Systems Engineering Guide for SoS [5], an SoS can be classified according to the way it is managed and its openness to change and new capabilities:

- Virtual SoS: in this type of SoS, there is a no central management authority. Large-scale behavior may emerge. This type of SoS must rely on invisible mechanisms for maintenance.

- Collaborative SoS: the component systems interact (more or less voluntarily) to reach agreed upon central goals. The central players collectively decide how to provide or deny service, thereby providing some means of enforcing and maintaining standards.

- Acknowledged SoS in this type of SoS, changes in the systems are based on collaboration between the SoS and the system. The SoS has recognized objectives, a designated manager, and resources for the SoS;

\footnotetext{
${ }^{3}$ https://imindmap.com.

${ }^{4} \mathrm{https}: / / \mathrm{www} \cdot \mathrm{mindmeister.com} / \mathrm{fr}$
} 
however, the constituent systems retain their independent ownership, objectives, and development approaches.

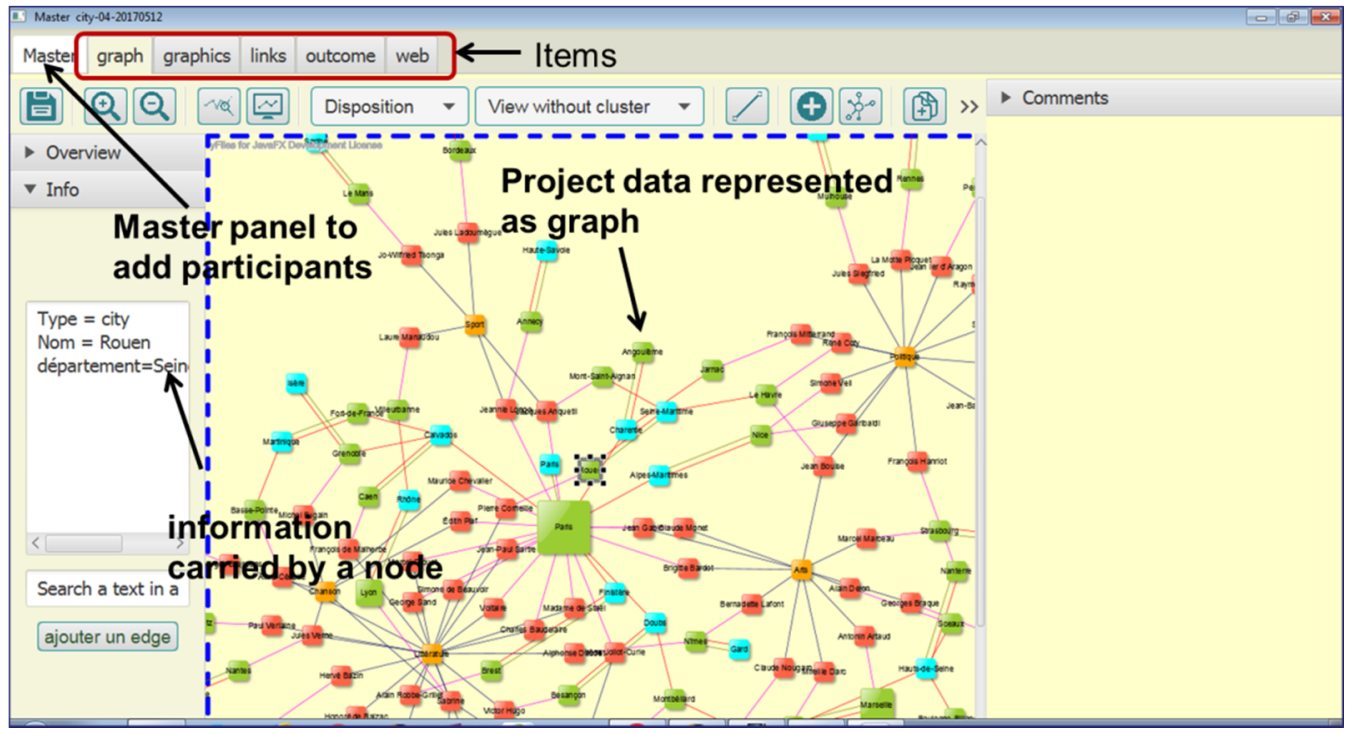

Figure 1: The main module of the ECOPACK brainstorming platform

- Directed SoS are those in which the integrated systemof-systems is built and managed to reach specific purposes. It is centrally managed during long-term operation to continue to fulfill those purposes as well as any new ones the system owners might wish to address. The component systems maintain an ability to operate independently, but their normal operational mode is subordinated to the central managed purpose.

SoS approach promotes a new way of thinking for solving unprecedented and complex challenges. It does not impose specific tools, methods, or practices; instead, the development and maintenance of such systems relies on principles cited above. These principles serve the purpose of providing a unifying guide to define SoS architecture and identify the interfaces between the systems. The interfaces are the connective points that enable the individual systems to interoperate as a whole system. The maintenance of the interfaces enables the constituent systems to evolve while remaining in and contributing to the integrated whole.

We claim that the SoS approach is an adequate solution to resolve resources capitalization of independent systems.

\section{OUR PROPOSAL}

This paper addresses the question of capitalization resources of collaborative activities illustrated by the integration of ECOPACK platform and MEMORAeSoIS. In this section, we present first these two platforms then we present the interaction between ECOPACK and MEMORAeSoIS.

\subsection{ECOPACK brainstorming platform}

ECOPACK is a brainstorming digital platform that has been developed to meet the needs of ideation, innovation and strategic analysis of experts groups. This digital platform follows the knowledge ecosystem vision that fosters the dynamic evolution of knowledge interactions between users in order to enhance decision-making and innovation. Technically, ECOPACK platform aims at:

- providing a multi-user platform that support several devices (tablets, smartphones, personal computer, etc.) to allow different forms of collaboration;

- defining collaborative applications where each user benefits from different types of interaction and activity and is able to exploit his/her own resources to collaborate.

ECOPACK manages a set of projects where different types of data are grouped as items, presented and gathered by a main module (Fig.1). One of the originality of ECOPACK is that it provides a dynamic data representation as a graph, which facilitates their interpretation by experts. The graph can be shown in different ways following several types of graph drawing algorithms, including those that create hierarchical, organic, orthogonal and circular layout. Indeed, the different ways of visualizing a graph provide different insights, and hidden relationships and interesting patterns are revealed. This can help to make relevant observations, to carry out a pertinent strategic analysis, etc. Also, there is the ability to zoom in and out with the graph. In addition, the main module provides the possibility to create clusters within the graph. Clusters can be modified, and nodes can be added or removed from clusters. Cluster view also can be toggled on or off to show the graph with or without clusters based on the course of discussion taking place. Participants can create notes related to certain aspect of the graph shown in the main module. The creation of notes is handled by a separate dedicated application that resides within the participant's device. Notes are then sent to the main module to be shown in the graph for everyone to see and discuss. Furthermore, notes can 
target the graph as a whole, or a specific part of the graph. It also can target a node or an edge, or even a group of nodes and edges combined together as a cluster. Another feature is the dashboard of experts' activities with the platform. This is useful to give precise statistical information of experts' contributions.

ECOPACK platform provides thus several functionalities allowing participants to accomplish a brainstorming session or conduct a strategic analysis session based on data presented in the form of a map. In addition, ECOPACK allow users keep track of the results of their activities by the mean of automatic reports.

\subsection{MEMORAeSoIS platform}

MEMORAeSoIS platform [10] was developed to meet the need of accessing, sharing and capitalizing resources coming from independent and autonomous systems. MEMORAeSoIS is based on MEMORAe model [1]. Therefore, before given more details about MEMORAeSoIS we introduce first MEMORAe approach. The MEMORAe approach is the combination of a model and a web platform to manage heterogeneous knowledge in an organization. It is based on OWL language and semantic web standards (FOAF, SIOC, BIBO). Users in MEMORAe platform are given access to several knowledge bases through a semantic map. The later describes the knowledge in the base by the mean of shared terminology in the organization. The main advantage of this approach is that it allows indexing all types of resources around a semantic map. Resources are defined as "information vectors" and are distinguished according to whether they are simple or complex. A simple resource is a whole. A document, a note are examples of simple resources. Complex resources consist of other resources such as a wiki or an agenda. In this way, MEMORAe takes into account documentary resources and social resources. MEMORAe platform allow thus users managing various types of resources. However, all of them are native of the platform. To give users the possibility to access and capitalize resources created by external information systems, MEMORAeSoIS platform was proposed based on the capabilities of MEMORAe approaches (indexing, annotating, sharing and tracing the resources).

MEMORAeSoIS architecture is a "Leader/Follower" architecture. That is, MEMORAe platform is the leader system responsible for the orchestration of SoIS as a knowledge base serving all other Information Systems. Each follower system work independently and has its own services/functions and databases. So, while some systems are openly providing an API for requesting their services, other systems are closed and operate as black boxes. Information can be represented in different ways within different systems, thus, the SoIS might have trouble access information, if the services of that system are not available through an API. Therefore, to solve this interoperability issue, two methods exists [3]: (i) creating a software model of each system, to collects data from the system and generates the outputs. (ii) creating a high level common language to describe data, where each system can represent its data such that other systems may interpret. Creating a common language is a complex task and requires knowing the information model of each system. In
MEMORAeSoIS authors have opted for the first solution. Resources of the external Information System is made available to the user by means of the data wrapper and a server/observer (details are presented in [10]). It is necessary, however, that each system provides an API allowing an external system to interact with it. The ideas present in this architecture of SoIS can be summarized in the following list:

- The user is provided with access to several Information Systems.

- The user can choose which Information System(s) he/she would like to connect to. After connecting to various systems of choice, the user can access resources and services in their respective environment.

- It is then possible to work with resources produced by different Information Systems from within the SoIS.

- The resources produced by different Information Systems are managed in the SoIS by the means of the services provided by the leader system.

- Using the services of the leader system as a way to manage knowledge with the SoIS will enable the user from indexing, sharing and tracing resources within the SoIS.

\subsection{Use case}

To illustrate our proposal, we consider the scenario of the challenges of naval defense of France. More precisely, we focus on a question proposed by the regional association AR24 Picardie of the Institute for Higher National Defense Studies (IHEDN): how to strengthen France' defense strategy? To answer this question, brainstorming sessions (using ECOPACK) was conducted from a database on the relationships between on the one hand companies, suppliers, buyers and financiers of military naval equipment and on the other hand armed conflicts and diplomatic meetings. Thanks to the functionalities provided by ECOPACK (ex. the possibility to represent data by a graph) participants were able to make some observations that allowed them to make hypothesis to improve the defense strategy. However, information available in ECOPACK were not sufficient to answer the question effectively. Indeed, the defense strategy is obviously largely influenced by the global political and economic context. For example, a country that is involved in a conflict may need armaments to defend and consequently influence France's defense strategy. Users in a brainstorming session about defense strategy may need more information about a conflict in a client country. Some of the information concerning political and economic context can be found in google search. A user may thus make a search google and decide to share the information that judges relevant with his colleagues. This information may be relevant to evaluate the current defense strategy of a country regarding the evolution of the political and economic context. Consequently, the user's observation and commentary made in a brainstorming session may be influenced by different information coming from other "system/web". 


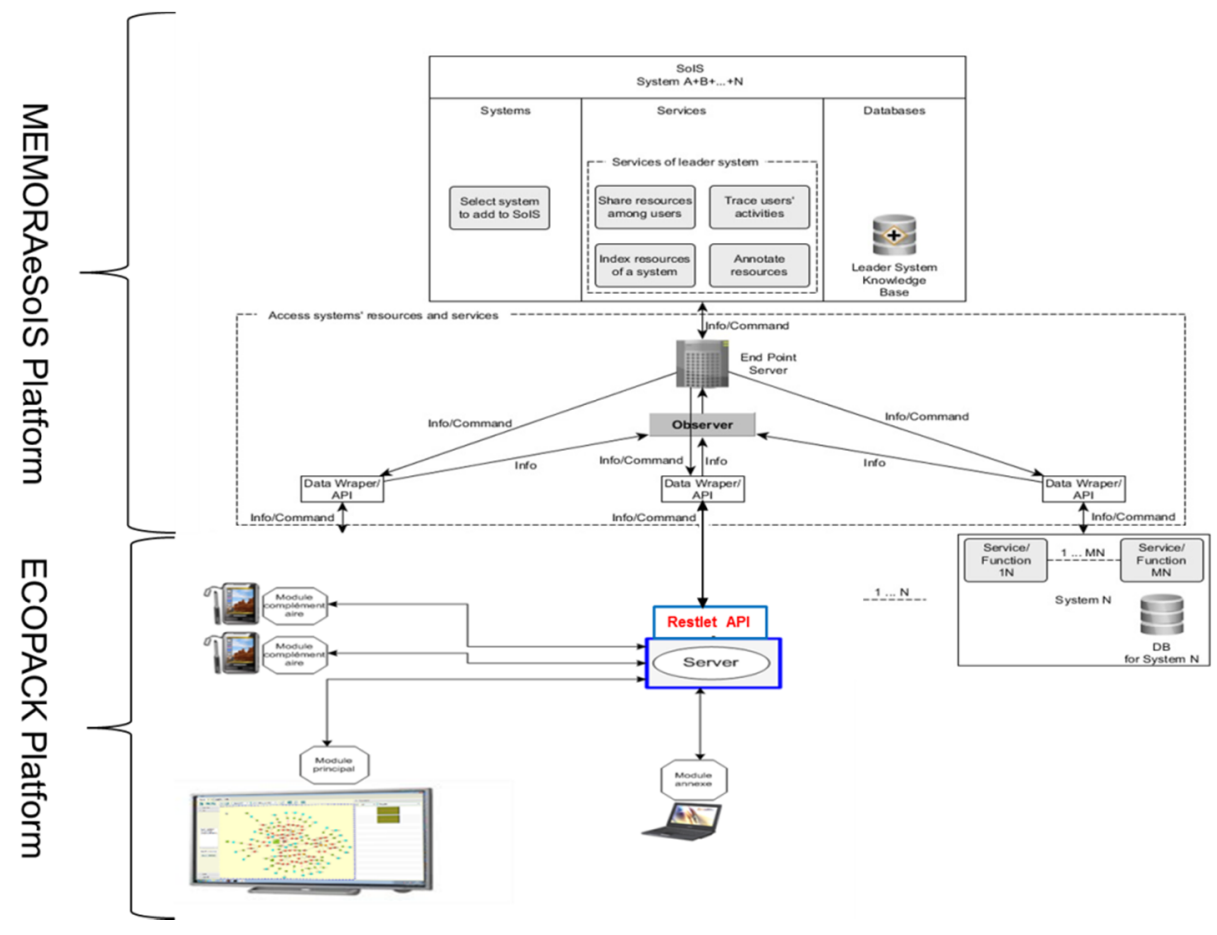

Figure 2: Integration of ECOPACK in MEMORAESoIS.

It is primordial thus to not only keep track of information and knowledge generated during a brainstorming session but also to keep track of the different information coming from other systems used by users in the brainstorming session.

For the management level, information flow can be optimized if all resources are available in a centralized access point that can reference those resources from their original information systems. In the next section, we show how all resources useful for collaborative activities can be access and indexed from a single point.

\subsection{Interaction of MEMORAe SoIS and ECOPACK}

According to Salah et al. [10], to integrate a new system in MEMORAeSoIS, it is necessary to develop a new module that collects data from the target system and generates the outputs. Figure 2, shows the interaction between MEMORAeSoIS and ECOPACK. Data wrapper is responsible on collecting data from external systems while server/observer ensure the interaction with the leader system "MEMORAE platform". Also, as mentioned in section 3.2, to be able to interact with an external system from MEMORAeSoIS the target system must provide an API for requesting their services. The initial version of ECOPACK does not provide an API allowing an external system to interact with it.The first step to allow MAMEOAeSoIS interacting with ECOPACK and thus capitalizing heterogeneous resources was to develop an API. The second step consists on developing the data wrapper in MEMORAeSoIs to collect data from ECOPACK.
Note that, once the data wrapper is developed all the functionalities to manage resources are already available in MEMORAeSoIS. Consequently, adding a new system to MEMORAeSoIS does not necessitate important development. ECOPACK platform was developed based on several pertinent technologies such as Akka5 and Restlet6 framework. Akka is a toolkit for building highly concurrent, distributed and resilient message-driven applications for Java and Scala. Restlet is an open source RESTful web API framework for the Java platform. It supports several data format, Internet transport and service description standards like HTTP, XML, JSON. So, to reach our goal we have developed a Restlet API to allow technical interoperability between ECOPACK and MEMORAeSoIS. This API allows accessing the resources stored in ECOPACK. Resources in ECOPACK are organized in projects and projects version. A Resource can be a brainstorming report, a comment, a note, a graph, etc. Note that in MEMORAeSoIS system resources of its components systems does not reside in the SoIS, but rather are referenced from the Information Systems comprising it.

In figure 3, we can see that ECOPACK is accessible from MEMORAeSoIS as well as other systems that already existed in the SoIS: Tiddlywiki, Twitter, Google search, Google contact, Microsoft OneNote and two PLM (Product Lifecycle Management) systems (OdooERP and ARAS Innovator).

\footnotetext{
${ }^{5}$ https://akka.io/

${ }^{6}$ https://restlet.com
} 


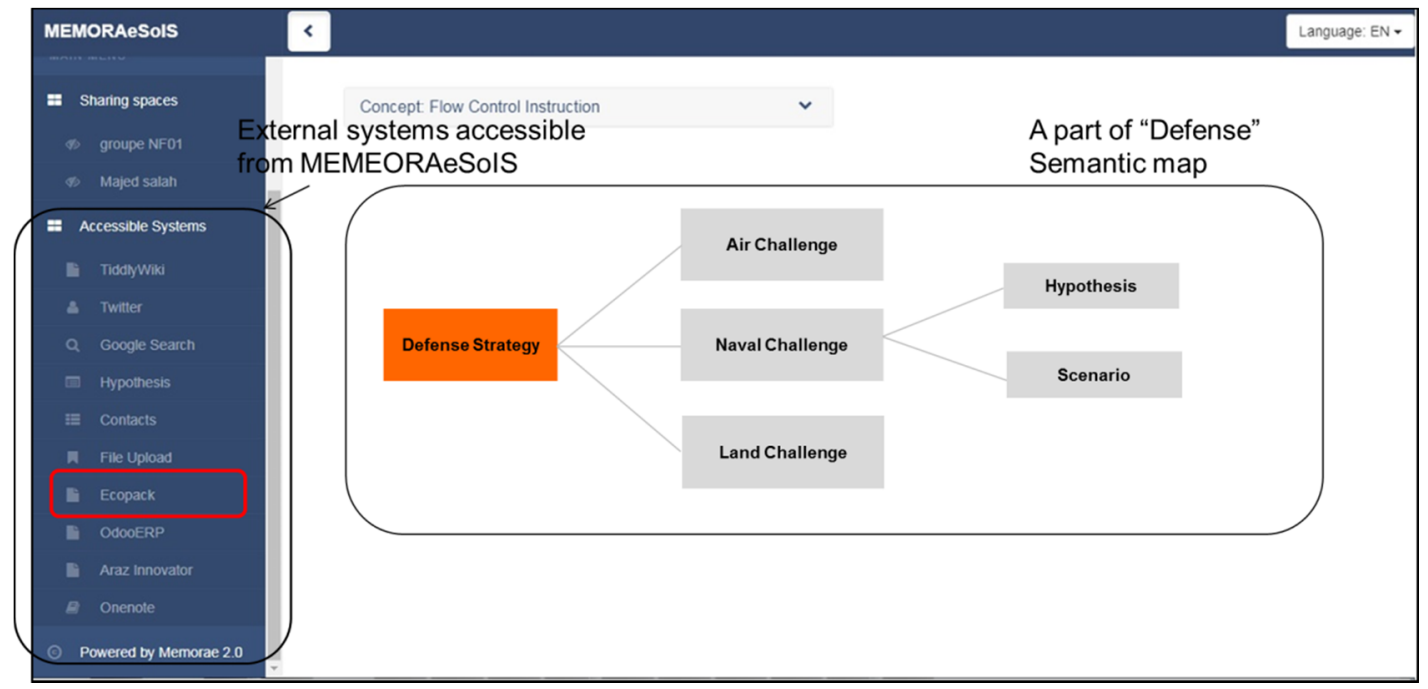

Figure 3: Systems accessible in MEMORAeSoIS.

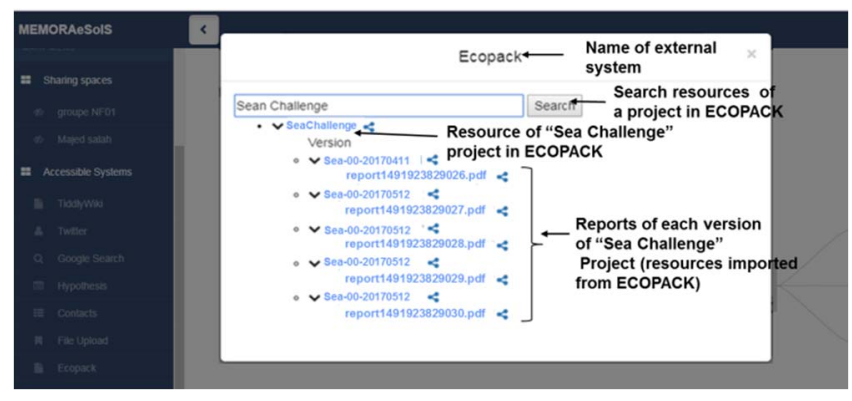

Figure 4: Panel to access resources available in ECOPACK.

We can also see a part of the semantic map describing the global domain of defense strategy. The concepts of this map allow indexing resources collected from ECOPACK and other systems. In figure 4, we see a part of ECOPACK resources that are imported in MEMORAeSoIS namely a set of reports concerning the "Sea Challenge" project and its versions. Figure 5 shows a set of additional resources concerning the challenges of naval defense of France.

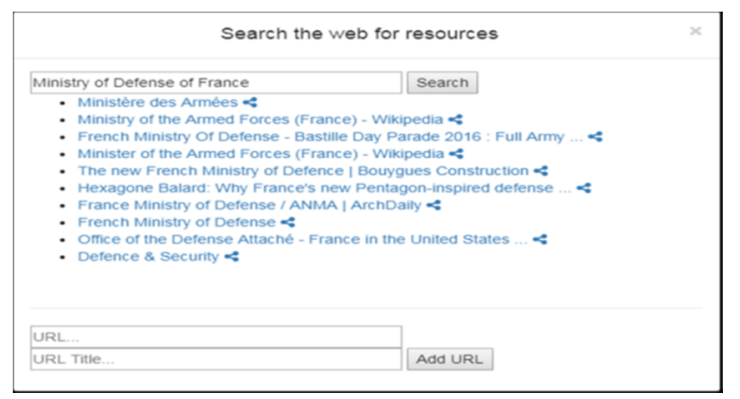

Figure 5: Panel to access resources available in Google search.
Indeed, in figure 5 we can see a set of websites that addresses the subject of defense in France. Using the box of "Google Search" (accessible in MEMORAeSoIS) users can make a search on the web and capitalize all relevant websites links. All these resources that are interesting for the topic of France's naval defense can now be indexed and thus capitalized by the adequate concept in the semantic map. Figure 6 shows a box to choose a concept of semantic map (Naval Challenge) to index resources of ECOPACK (reports of "Sea Challenge").

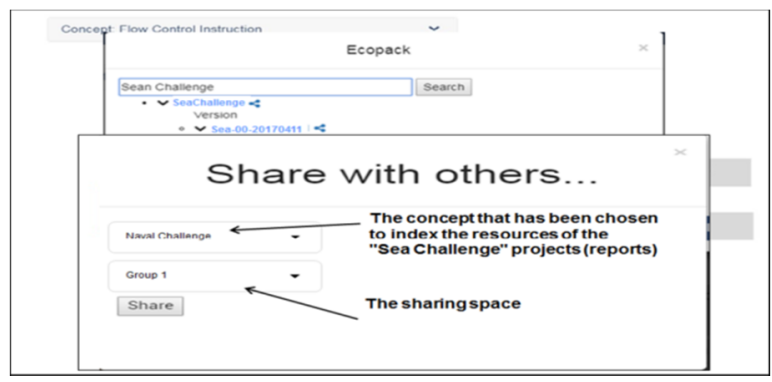

Figure 6: Indexing and sharing resources.

\section{CONCLUSIONS}

The goal of this paper was to provide a solution to capitalize resources of collaborative activities, especially in the context of brainstorming activities. We have showed that in brainstorming activities participants use several Information System and Web tools to gather information about the topic of brainstorming. This raised the question of managing heterogeneous resources coming from independent and autonomous systems. To solve this issue, we have opted to use MEMORAeSoIS platform. Thanks to the flexibility of the architecture of this platform, we have integrated easily ECOPACK to this SoIS platform. This by developing an API to interact with ECOPACK and a data wrapper allowing 
MEMORAeSoIS to collect resources from ECOPACK. The most important value for the collaborative SoIS is in its ability to trace the result of users' activities. That is, all resources generated while using ECOPACK and all external resources that are useful to accomplish an activity in ECOPACK. In addition, thanks to the semantic map of MEMORAeSoIS, all users have a common understanding of the resources available in the SoIS.

The next step is to expand our work by developing software allowing export resources from MEMORAeSoIS to ECOPACK in order to reuse them. Furthermore, MEMORAeSoIS offers the possibility to access several useful systems and thus to collect various resources from different systems that may be useful for a brainstorming session. It is interesting thus to provide ECOPACK users the possibility to get these resources from MEMORAeSoIS. Indeed, this may be done by exporting resources from MEMORAeSoIS to ECOPACK and make them available through an item in ECOPACK (ex. a link of a website). Also we plan to develop a recommendation system allowing MEMORAeSoIS users discovering useful resources indexed by the concept of other semantic map. This can be done using matching ontologies algorithms. Indeed, matching ontologies algorithms help to discover the equivalent concept between two or several ontologies. This kind of algorithms may be useful to discover equivalent concepts between two or several semantic map and thus to recommend useful resources to users.

\section{ACKNOWLEDGMENTS}

This project was carried out both under ECOPACK project (funded by ANR-ASTRID program) and in the framework of the Labex MS2T, which was funded by the French Government, through the program "Investments for the future" managed by the National Agency for Research (Reference ANR-11-IDEX-000402).

\section{REFERENCES}

[1] Abel, M-H., 2008. Competencies Management and Learning Organizational Memory. Journal of Knowledge Management: special issue on Competencies management: Integrat-ing Semantic Web and Technology Enhanced Learning Approaches for Effective Knowledge Management, 12, pp. 15-30.

[2] Boardman, J., Sauser, B., 2006. System of Systems - the meaning of Of, IEEE/SMC International Conference on System of Systems Engineering, pp. 118123.

[3] Bowen, R. M. and Sahin, F., 2010. A net-centric xml based system of systems architecture for human tracking, in System of Systems Engineering (SoSE), IEEE International Conference, pp. 1-6.

[4] Carney, D., Fisher, D., Place, P., 2005. Topics in Interoperability: System-ofSystems Evolution. Software Engineering Institute, Carnegie Mellon University, Pittsburgh.

[5] Department of Defense., 2004. Defense Acquisition Guidebook Ch. 4 "System of Systems Engineering," Washington, DC: Pentagon.

[6] Maier, M.W., 1998. Architecting principles for systems-of-systems, Systems Engineering, 1(4), pp. 267-284.

[7] Moulin, C., Kaeri, Y., Sugawara, K., Abel, M.H., 2016. Capitalization of remote collaborative brainstorming activities, Computer Standards \& Interfaces, 48, pp. 217 224.

[8] Osborn, A. F. 1953. Applied Imagination. New York: Scribners.
[9] Popper, S., Bankes, S., Callaway, R., and DeLaurentis, D., 2004. System-ofSystems Symposium: Report on a Summer Conversation, Potomac Institute for Policy Studies, Arlington, VA.

[10] Salah, M. Misséri V., Abel, M.H, 2016. Managing Heterogeneous Information in a System of Information Systems. 8th International Conference on Knowledge Management and Information Sharing, Porto, Portugal.

[11] Selberg, S. A., \& Austin, M. A. 2008. Toward an Evolutionary System of Systems Architecture. In INCOSE International Symposium, 18(1), pp. 1065-1078. 